\title{
Electrical Control in Neurons by the Ketogenic Diet
}

\author{
Nagisa Sada ${ }^{1,2}$ and Tsuyoshi Inoue ${ }^{1 *}$ \\ ${ }^{1}$ Department of Biophysical Chemistry, Graduate School of Medicine, Dentistry and Pharmaceutical Sciences, Okayama \\ University, Okayama, Japan, 'Department of Hygiene, Kawasaki Medical School, Kurashiki, Japan
}

The ketogenic diet is used as a diet treatment for drug-resistant epilepsy, but there are no antiepileptic drugs based on the ketogenic diet. The ketogenic diet changes energy metabolites (ketone bodies, glucose and lactate) in the brain, which consequently changes electrical activities in neurons and ultimately suppresses seizures in epileptic patients. In order to elucidate the antiseizure effects of the ketogenic diet, it is important to clarify the mechanism by which these metabolic changes are converted to electrical changes in neurons. In this review, we summarize electrophysiological studies focusing on electrical control in neurons by the ketogenic diet. Recent studies have identified electrical regulators driven by the ketogenic diet: ion channels (ATP-sensitive $\mathrm{K}^{+}$channels and voltage-dependent $\mathrm{Ca}^{2+}$ channels), synaptic receptors (AMPA-type glutamate receptors and adenosine $A_{1}$ receptors), neurotransmitter transporters (vesicular glutamate transporters), and others (BCL-2associated agonist of cell death and lactate dehydrogenase). Thus, the ketogenic diet presumably elicits neuronal inhibition via the combined actions of these molecules. From the viewpoint of drug development, these molecules are valuable as targets for the

OPEN ACCESS

Edited by: Li-Rong Shao, Johns Hopkins University, United States

Reviewed by: Susan A. Masino, Trinity College, United States Masahito Kawamura, Jikei University School of Medicine, Japan

*Correspondence: Tsuyoshi Inoue tinoue@okayama-u.ac.jp

Received: 30 April 2018 Accepted: 26 June 2018 Published: 16 July 2018

Citation: Sada N and Inoue T (2018) Electrical Control in Neurons by the Ketogenic Diet.

Front. Cell. Neurosci. 12:208. doi: 10.3389/fncel.2018.00208 development of new antiepileptic drugs. Drug therapy to mimic the ketogenic diet may be feasible in the future, through the combination of multiple antiepileptic drugs targeting these molecules.

Keywords: ketogenic diet, ketone body, glucose, lactate, epilepsy, antiepileptic drug, electrophysiology

\section{INTRODUCTION}

Neurons, which are connected by synapses, exhibit electrical activities in the living brain. The electrical activities in neurons are generated by ion channels and synaptic receptors. Although these electrical activities are essential for normal brain function, they become excessive in the brains of epileptic patients. Therefore, medicines for epilepsy have to suppress the neuronal hyperexcitation, and currently-used antiepileptic drugs have been designed to act on ion channels and synaptic receptors (Meldrum and Rogawski, 2007). For example, carbamazepine and lamotrigine inhibit voltage-dependent $\mathrm{Na}^{+}$channels, gabapentin inhibits the $\alpha 2 \delta$ subunits of L-type $\mathrm{Ca}^{2+}$ channels, phenobarbital activates $\mathrm{GABA}_{\mathrm{A}}$ receptors, and perampanel modulates AMPA-type glutamate receptors (reviewed in Bialer and White, 2010).

However, these antiepileptic drugs are not effective for all epileptic patients. Approximately $1 \%$ of the world's population suffer from epilepsy, and one-third of epileptic patients are resistant to currently-available antiepileptic drugs (Kwan and Brodie, 2000). It should be noted that the diet treatment using the ketogenic diet is effective for some patients with drug-resistant epilepsy (Freeman et al., 1998; Neal et al., 2008). However, since the ketogenic diet 
is an unbalanced diet consisting of high-fat and lowcarbohydrate, new medicines based on the ketogenic diet will be useful for the treatment of drug-resistant epilepsy. To address this issue, recent studies have elucidated the antiseizure mechanisms of the ketogenic diet at the molecular level (reviewed in Lutas and Yellen, 2013; Boison, 2017; Rho, 2017; Simeone et al., 2018). Since these reviews have introduced many antiseizure mechanisms associated with the metabolic, electrical, epigenetic and inflammatory changes induced by the ketogenic diet, we do not summarize these mechanisms thoroughly in the present review. We here summarize electrophysiological studies that focus on electrical control in neurons by the ketogenic diet.

From the viewpoint of drug development, the molecules involved in the ketogenic diet are valuable as "target molecule" to explore candidates of new antiepileptic drugs. Toward drug development, we then identify small chemical compounds acting on the target molecule, called as "seed compound" for screening from compound libraries. We finally identify drug candidates by compound screening using the target molecule and seed compound. Thus, the identification of molecules involved in the ketogenic diet is the first step toward the development of new antiepileptic drugs for drug-resistant epilepsy.

\section{KETOGENIC DIET FOR DRUG-RESISTANT EPILEPSY}

The ketogenic diet treatment was developed in the 1920s, and its concept dates back to biblical times (Wheless, 2008). The diet treatment was originally developed by Dr. Wilder at the Mayo Clinic in 1921, and its modified version using a medium-chain triglyceride (MCT) diet was developed in the 1970s (Huttenlocher et al., 1971). These ketogenic diets are high-fat and low-carbohydrate diets, which produce ketone bodies ( $\beta$-hydroxybutyrate and acetoacetate) by the liver. Ketone bodies are then delivered to the brain and used as alternative energy sources to glucose. The ketogenic diet not only increases ketone bodies, but also mildly decreases blood glucose levels in epileptic patients (Huttenlocher, 1976). These two metabolic changes are now recognized to exert antiseizure effects (reviewed in Rho, 2017). The direct actions of ketone bodies were recently reviewed in detail (Simeone et al., 2018).

Historically, although the ketogenic diet treatment was developed in 1921, its use in clinical setting decreased after the development of the antiepileptic drug diphenylhydantoin (phenytoin) in 1938 (Merritt and Putnam, 1938). However, the diet treatment re-attracted attention in the 1990s because it was shown to be effective for patients with drug-resistant epilepsy (Freeman et al., 1998). The ketogenic diet treatment is now used in many countries worldwide (Kossoff and McGrogan, 2005).

The efficacy of the ketogenic diet for drug-resistant epilepsy has been confirmed in many clinical studies. Randomized clinical trials revealed that approximately $40 \%$ of pediatric patients with drug-resistant epilepsy were controlled by the ketogenic diet (Neal et al., 2008) and that the efficacy was similar between the classical and MCT versions of the ketogenic diet (Neal et al., 2009). The ketogenic diet was effective for patients with Dravet syndrome (Caraballo et al., 2005), a severe childhood epilepsy with high mortality (Sakauchi et al., 2011). In addition to childhood epilepsy, growing evidence supports that the ketogenic diet is also effective for adult patients with intractable epilepsy (Klein et al., 2014; Liu et al., 2018).

\section{MOLECULES FOR ELECTRICAL CONTROL BY THE KETOGENIC DIET}

As described above, the ketogenic diet is now established as a treatment for patients with drug-resistant epilepsy. Since seizures are elicited by the hyperexcitation of electrical activities in neurons, it is mechanistically presumed that the ketogenic diet acts on the molecules generating electrical currents, such as ion channels and synaptic receptors. This issue can be directly addressed using electrophysiological techniques in rodents. We therefore introduce electrophysiology-based studies, in which the following seven molecules have been identified as regulators of neuronal electrical activities by the ketogenic diet (Figure 1).

\section{ATP-Sensitive $\mathrm{K}^{+}$Channels}

The ketogenic diet consists of high-fat and low-carbohydrate, and mainly elicits two metabolic changes in blood: increases in ketone bodies and decreases in glucose (Bough et al., 2006). In rodent studies, the ketogenic diet was shown to increase the blood plasma level of $\beta$-hydroxybutyrate to 1-8 $\mathrm{mM}$ (Bough and Eagles, 1999; Bough et al., 1999, 2006), which is similar to that in humans (Neal et al., 2009). Using electrophysiological techniques, Yellen and colleagues found adenosine 5 '-triphosphate (ATP)-sensitive $\mathrm{K}^{+}$channels $\left(\mathrm{K}_{\mathrm{ATP}}\right.$ channels) as the molecule that ketone bodies acted on (Ma et al., 2007). K $\mathrm{K}_{\mathrm{ATP}}$ channels are known to be metabolic sensors that regulate electrical activities, which are blocked by intracellular ATP. Ma et al. (2007) proposed that ketone bodies increase intracellular global ATP, but decrease glycolytic ATP production, which leads to decreases in ATP near the plasma membrane, the opening of $\mathrm{K}_{\mathrm{ATP}}$ channels, and reductions in the firing rate of neurons. Consistent with this, the opening of $\mathrm{K}_{\mathrm{ATP}}$ channels by ketone bodies was directly demonstrated by single channel recordings (Tanner et al., 2011).

\section{Voltage-Dependent $\mathrm{Ca}^{2+}$ Channels}

Voltage-dependent $\mathrm{Ca}^{2+}$ channels (VDCCs) in presynaptic terminals are known to be essential for synaptic transmission (Zucker and Regehr, 2002; Catterall et al., 2013). By using patch-clamp recordings from hippocampal slices, Inoue and colleagues demonstrated that acetoacetate inhibited $\mathrm{Ca}^{2+}$ influx through VDCCs in CA1 pyramidal cells (Kadowaki et al., 2017). They also found that acetoacetate modulated the short-term synaptic plasticity of excitatory postsynaptic currents (EPSCs) in pyramidal cells, showing that it acted on presynaptic VDCCs. Interestingly, acetoacetate reduced EPSCs in slices exhibiting epileptiform activities, but not in normal slices (Kadowaki et al., 2017), suggesting that the effects of acetoacetate preferably emerge in the hyperexcitable state of the brain. Although its VDCC types remain unclear, it is probably mediated 


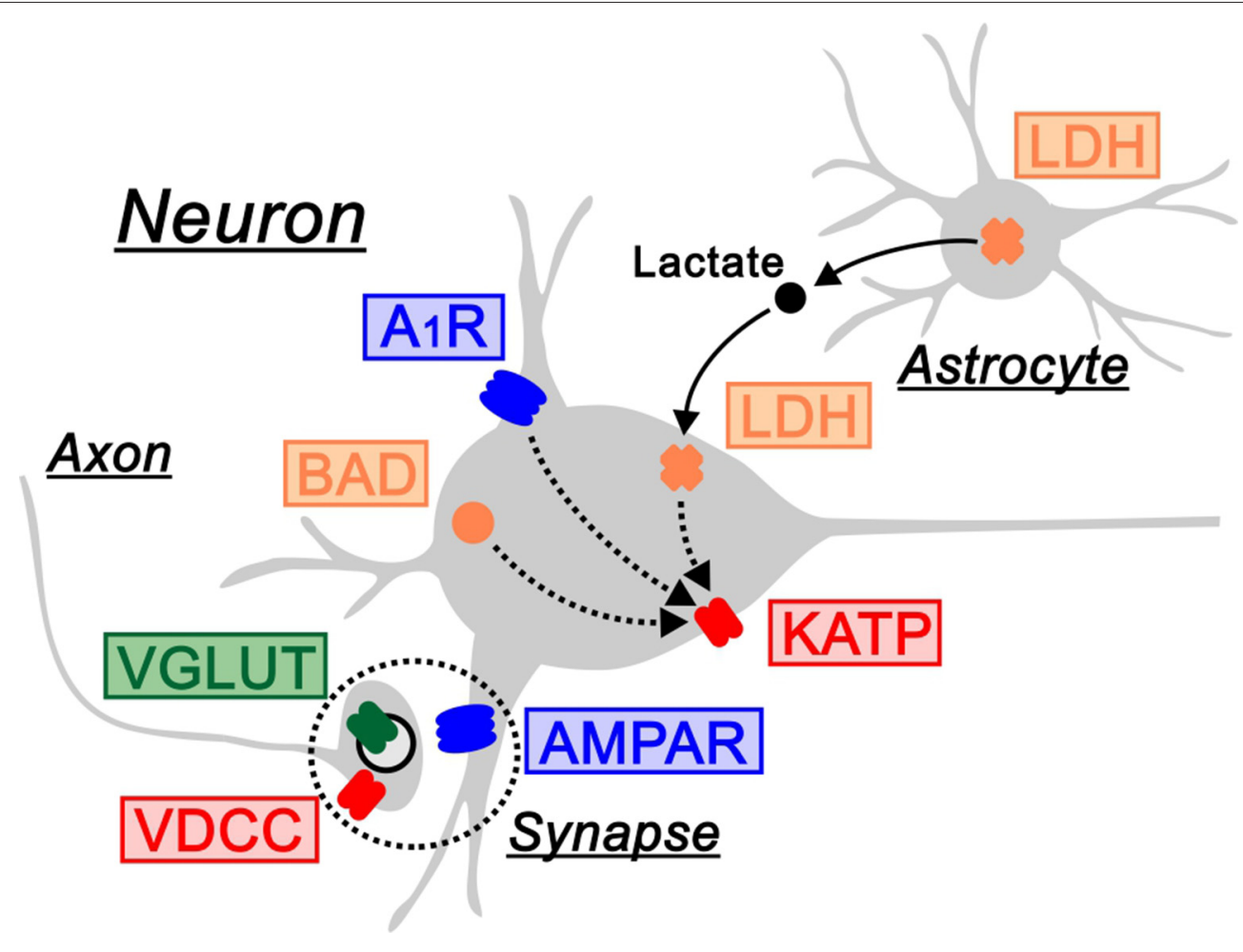

FIGURE 1 | Electrical regulators driven by the ketogenic diet. The ketogenic diet is associated with the following molecules that regulate electrical activities in neurons: ATP-sensitive $\mathrm{K}^{+}$channels (KATP) and voltage-dependent $\mathrm{Ca}^{2+}$ channels (VDCC) as ion channels in red, AMPA-type glutamate receptors (AMPAR) and adenosine $A_{1}$ receptors $\left(A_{1} R\right)$ as synaptic receptors in blue, vesicular glutamate transporters (VGLUT) as neurotransmitter transporters in green, and $B C L-2$-associated agonist of cell death (BAD) and lactate dehydrogenase (LDH) as other molecules in orange. $\mathrm{LDH}$ is located in the astrocyte-neuron lactate shuttle (arrows). $A_{1} R, B A D$ and LDH regulate electrical activities via $K_{\text {ATP }}$ channels (dotted arrows). Synapse is indicated by dotted circles.

by P/Q-type (Cav2.1) or N-type (Cav2.2) $\mathrm{Ca}^{2+}$ channels, because these $\mathrm{Ca}^{2+}$ channels mainly contribute to synaptic transmission (Catterall et al., 2013). A previous study reported that $\beta$-hydroxybutyrate inhibited $\mathrm{N}$-type $\mathrm{Ca}^{2+}$ channels in rat sympathetic neurons of the peripheral nervous system (Won et al., 2013).

\section{Vesicular Glutamate Transporters}

Vesicular glutamate transporters (VGLUTs), which fill synaptic vesicles with glutamate, critically regulate excitatory synaptic transmission in the brain (Fremeau et al., 2004). Thus, VGLUTs are also electrical regulators that indirectly affect postsynaptic glutamate receptors. Using a proteoliposome containing purified VGLUTs, Moriyama and colleagues demonstrated that acetoacetate was a specific inhibitor of VGLUTs (Juge et al., 2010). Although VGLUTs were activated by $\mathrm{Cl}^{-}$ions, the elevated VGLUT activities were inhibited by acetoacetate: therefore, they proposed that VGLUTs are oppositely regulated by $\mathrm{Cl}^{-}$ions and acetoacetate. Functionally, acetoacetate inhibited miniature EPSCs in CA1 pyramidal cells of hippocampal slices and suppressed acute seizures in rats in vivo (Juge et al., 2010).

\section{Adenosine $A_{1}$ Receptors}

Adenosine is an inhibitory neuromodulator, which acts on four types of adenosine receptors in the brain $\left(A_{1}, A_{2 A}, A_{2 B}\right.$ and $\mathrm{A}_{3}$; Dunwiddie and Masino, 2001). Among them, the activation of adenosine $A_{1}$ receptors $\left(A_{1} R s\right)$ was shown to suppress chronic seizures in a mouse model of pharmacoresistant mesial temporal lobe epilepsy (Gouder et al., 2003). Boison and colleagues found that the antiseizure effects of the ketogenic diet were not observed in knockout mice of adenosine $A_{1}$ Rs (Masino et al., 2011). Furthermore, the antiseizure mechanism is presumably attributed to the decreases in glucose by the ketogenic diet. By using patch-clamp recordings from hippocampal slices, Masino and colleagues revealed that the decreases in glucose hyperpolarized hippocampal pyramidal cells, and this was mediated by adenosine $\mathrm{A}_{1}$ Rs and $\mathrm{K}_{\mathrm{ATP}}$ channels (Kawamura et al., 2010). They further found that the decreases in glucose reduced the neural excitability in mice fed the ketogenic diet, and this was also mediated by adenosine $\mathrm{A}_{1} \mathrm{Rs}$ and $\mathrm{K}_{\mathrm{ATP}}$ channels (Kawamura et al., 2014).

\section{AMPA-Type Glutamate Receptors}

The MCT ketogenic diet is a modified version of the classical ketogenic diet (Huttenlocher et al., 1971), and increases not only ketone bodies but also two fatty acids (octanoic and decanoic acids) in the blood of epileptic patients (Haidukewych et al., 1982; Sills et al., 1986). Octanoic and decanoic acids are straightchain saturated monocarboxylic acids with 8 and 10 carbons, 
respectively. These medium-chain fatty acids directly penetrate the blood-brain barrier (Oldendorf, 1973). Walker, Williams, and colleagues reported that decanoic acid, but not octanoic acid, suppressed in vitro epileptiform activity in entorhinal cortexhippocampus slices (Chang et al., 2013). They also elucidated the underlying mechanism, in which decanoic acid reduced EPSCs via AMPA-type glutamate receptors (Chang et al., 2016). By using electrophysiological recordings from an oocyte expression system, they showed that decanoic acid inhibited glutamateinduced currents derived from various types of AMPA receptor subunits (GluA1, GluA1/2 and GluA2/3), and also showed that it was the most sensitive to GluA2/3 (Chang et al., 2016).

\section{BCL-2-Associated Agonist of Cell Death}

BCL-2-associated agonist of cell death (BAD) is a member of the BCL-2 family, which is known to regulate cellular metabolism (Giménez-Cassina and Danial, 2015). The knockout of BAD decreases the ability to use glucose and increases the ability to use $\beta$-hydroxybutyrate (Giménez-Cassina et al., 2012), which is similar to metabolism during the ketogenic diet (Bough et al., 2006). Yellen, Danial, and colleagues demonstrated that this metabolic switch by the BAD knockout protected against acute seizures in vivo (Giménez-Cassina et al., 2012). Chronic seizures in Kcna1-null mice, a mouse model of sudden unexpected death in epilepsy, were also suppressed by the BAD knockout (Foley et al., 2018), as well as by the ketogenic diet (Fenoglio-Simeone et al., 2009; Simeone et al., 2016).

From the viewpoint of electrical control, the antiseizure effects of the BAD knockout are mediated by $\mathrm{K}_{\mathrm{ATP}}$ channels (Giménez-Cassina et al., 2012; Martínez-François et al., 2018). $\mathrm{K}_{\mathrm{ATP}}$ channel currents in hippocampal neurons were shown to become larger in BAD-knockout mice, which was blocked by the genetic ablation of Kir6.2 (a pore-forming subunit of $\mathrm{K}_{\mathrm{ATP}}$ channels) or $\mathrm{K}_{\mathrm{ATP}}$ channel blockers (GiménezCassina et al., 2012). Consistent with this finding, the BAD knockout reduced neuronal excitability and epileptiform activity in brain slices, which was canceled by $\mathrm{K}_{\mathrm{ATP}}$ channel blockers (Martínez-François et al., 2018). The in vivo antiseizure effects of the BAD knockout were also canceled by the genetic ablation of Kir6.2 (Giménez-Cassina et al., 2012). Thus, the $\mathrm{BAD} \rightarrow \mathrm{K}_{\mathrm{ATP}}$ channel pathway regulates electrical and seizure control by the ketogenic diet. BAD is a unique molecule as electrical regulators by the ketogenic diet, because it mimics two metabolic changes during the ketogenic diet (decreases in glucose and increases in ketone bodies).

\section{Lactate Dehydrogenase}

Glucose is directly transported into neurons and used as an energy source. As an alternative metabolic pathway, glucose is transported into astrocytes and converted to lactate, which is then released to extracellular spaces and transported into neurons: this metabolic pathway is called the astrocyte-neuron lactate shuttle (Bélanger et al., 2011). Lactate is suggested to be a preferred energy source over glucose in the brain (Larrabee, 1995; Smith et al., 2003). This astrocyte-derived lactate regulates electrical activities in neurons (Rouach et al., 2008; Parsons and Hirasawa, 2010). Based on these backgrounds, Inoue and colleagues demonstrated that the astrocyte-neuron lactate shuttle contributes to neuronal inhibition and seizure suppression by the ketogenic diet (Sada et al., 2015). By using slice patch-clamp recordings, they found that ketogenic-like metabolic changes induced hyperpolarization in neurons, which was recovered by the activation of the lactate shuttle. They also found that the inhibition of lactate dehydrogenase (LDH), a metabolic enzyme located in the astrocyte-neuron lactate shuttle, induced hyperpolarization in neurons and suppressed chronic seizures in vivo in a mouse model of epilepsy (Sada et al., 2015).

From the viewpoint of electrical control, the neuronal hyperpolarization by the $\mathrm{LDH}$ inhibition is mediated by $\mathrm{K}_{\mathrm{ATP}}$ channels (Sada et al., 2015). They showed that the LDH inhibition induced hyperpolarization, which was recovered by $\mathrm{K}_{\mathrm{ATP}}$ channel blockers. This hyperpolarization was also recovered by pyruvate, a downstream metabolite of $\mathrm{LDH}$, indicating that it is mediated by pyruvate (Sada et al., 2015). Furthermore, this hyperpolarization was not recovered by an intracellular injection of ATP, suggesting that it is not dependent on ATP (Sada et al., 2015). Taken together, the $\mathrm{LDH} \rightarrow$ pyruvate $\rightarrow \mathrm{K}_{\mathrm{ATP}}$ channel pathway regulates electrical and seizure control by the ketogenic diet, which works in an energy-independent manner. $\mathrm{LDH}$ is the first metabolic enzyme identified as electrical regulators responsible for the ketogenic diet.

\section{DRUG DEVELOPMENT BASED ON THE KETOGENIC DIET}

The ketogenic diet treatment requires strict dietary control. Since medicines are markedly easier to administer, antiepileptic drugs based on the ketogenic diet will be useful for epileptic patients. Historically, the ketogenic diet treatment developed in the 1920s shifted to drug therapy using phenytoin in the late 1930s (Wheless, 2008).

Several approaches have been reported toward the development of ketogenic diet-based antiepileptic drugs. The first approach is ketone supplementation without dietary control. The oral administration of $R, S$-1,3-butanediol acetoacetate diester, a ketone ester, was shown to increase ketone bodies and decrease glucose in rat blood (D'Agostino et al., 2013; Kesl et al., 2016). These metabolic changes are similar to those observed during the ketogenic diet (Bough et al., 2006). Consistent with these findings, the ketone ester attenuated seizures in a mouse model of Angelman syndrome (Ciarlone et al., 2016) and in a rat model of absence epilepsy (Kovács et al., 2017). This ketone supplementation without dietary control may be useful as a new treatment for epilepsy that mimics the ketogenic diet.

The second approach is the new antiepileptic drug perampanel that was recently developed by a pharmaceutical company. It is designed to act on AMPA-type glutamate receptors (Hanada et al., 2011) and is effective for epileptic patients (French et al., 2012; Krauss et al., 2012). Different lines of basic studies by Walker, Williams, and colleagues demonstrated that decanoic acid, a metabolite of the MCT 
ketogenic diet, suppressed epileptiform activities via AMPA-type glutamate receptors (Chang et al., 2013, 2016). Thus, these two lines of evidence indicate that perampanel is a clinically-available drug to mimic the mechanism of action of the ketogenic diet.

The third approach originates from the antiepileptic drug stiripentol. Stiripentol has been clinically administrated to epileptic patients with Dravet syndrome (Chiron et al., 2000). It was originally shown to act on the GABAergic system in the brain (Trojnar et al., 2005; Quilichini et al., 2006). Inoue and colleagues reported that stiripentol inhibited $\mathrm{LDH}$, a metabolic enzyme responsible for the antiseizure effects of the ketogenic diet (Sada et al., 2015). They further identified a compound smaller than stiripentol, which retained the ability to inhibit LDH enzyme and suppress seizures in vivo. They proposed a strategy for drug development based on the ketogenic diet, that is, targeting LDH enzyme with stiripentol derivatives.

Interestingly, although $\mathrm{K}_{\mathrm{ATP}}$ channels are directly activated by ketone bodies (Ma et al., 2007; Tanner et al., 2011), it is also indirectly activated by BAD (Giménez-Cassina et al., 2012; Martínez-François et al., 2018) and LDH (Sada et al., 2015). $\mathrm{K}_{\mathrm{ATP}}$ channels are also involved in neuronal inhibition by adenosine $A_{1}$ Rs (Kawamura et al., 2010, 2014; Masino et al., 2011). Thus, $K_{\text {ATP }}$ channels are potential target molecules for drug development based on the ketogenic diet. However, it remains unclear whether $\mathrm{K}_{\mathrm{ATP}}$ channel blockers themselves are appropriate for the drug candidate. For example, $\mathrm{K}_{\mathrm{ATP}}$ channel blockers act on all types of neurons in the hippocampus (Zawar et al., 1999). In contrast, Sada et al. (2015) showed that the LDH inhibition was able to selectively hyperpolarize excitatory pyramidal cells but did not change inhibitory interneurons in the hippocampus.

Finally, some clinical studies have tested the feasibility of the ketogenic diet for the treatment of other neurological disorders. A clinical trial revealed that a modified version of the ketogenic diet improved core autism features in patients with autism spectrum disorder (Lee et al., 2018), and this was supported by animal studies (Ruskin et al., 2013, 2017). In addition, pilot clinical studies reported that the MCT ketogenic diet improved the cognitive performance of patients with Alzheimer's disease or mild cognitive impairment (Reger et al., 2004), and

\section{REFERENCES}

Bélanger, M., Allaman, I., and Magistretti, P. J. (2011). Brain energy metabolism: focus on astrocyte-neuron metabolic cooperation. Cell Metab. 14, 724-728. doi: 10.1016/j.cmet.2011.08.016

Bialer, M., and White, H. S. (2010). Key factors in the discovery and development of new antiepileptic drugs. Nat. Rev. Drug. Discov. 9, 68-82. doi: $10.1038 / \mathrm{nrd} 2997$

Boison, D. (2017). New insights into the mechanisms of the ketogenic diet. Curr. Opin. Neurol. 30, 187-192. doi: 10.1097/WCO.000000000 0000432

Bough, K. J., and Eagles, D. A. (1999). Ketogenic diet increases the resistance to pentylenetetrazole-induced seizures in the rat. Epilepsia 40, 138-143. doi: 10.1111/j.1528-1157.1999.tb02066.x

Bough, K. J., Valiyil, R., Han, F. T., and Eagles, D. A. (1999). Seizure resistance is dependent upon age and calorie restriction in rats fed a ketogenic diet. Epilepsy Res. 35, 21-28. doi: 10.1016/s0920-1211(98)00125-9 also reported that the ketogenic diet improved Parkinson's disease symptoms (Vanitallie et al., 2005). Although their efficacies have not fully evaluated in detail, further studies may potentially lead to drug development for these neurological disorders.

\section{CONCLUSION}

The ketogenic diet is effective for patients with drug-resistant epilepsy, and thus, these patients hunger for new antiepileptic drugs based on the ketogenic diet. Since seizures are elicited by the hyperexcitation of electrical activities in neurons, recent studies have uncovered the molecules responsible for the electrical control in neurons by the ketogenic diet. These electrical regulators are ion channels $\left(\mathrm{K}_{\mathrm{ATP}}\right.$ channels and VDCCs), synaptic receptors (AMPA-type glutamate receptors and adenosine $A_{1} R s$ ), neurotransmitter transporters (VGLUTs), and others (BAD and $\mathrm{LDH})$. Neuronal inhibition and seizure suppression by the ketogenic diet are presumed to be achieved by the combined actions of these molecules. Furthermore, some studies are now attempting to develop antiepileptic drugs targeting these molecules. Drug therapy to mimic the ketogenic diet will be possible in the future, through the combination of multiple antiepileptic drugs targeting these molecules.

\section{AUTHOR CONTRIBUTIONS}

NS and TI designed the idea and wrote and edited the manuscript.

\section{FUNDING}

NS is a research fellow of the Japan Society for the Promotion of Science, JSPS. This work was supported in part by Grant-inAid for Scientific Research (15K07966 and 18H02719 to TI) and Grant-in-Aid for JSPS Research Fellow (17J09536 to NS) from JSPS, the translational research program TR-SPRINT from the Japan Agency for Medical Research and Development, AMED (JP18lm0203019 to TI), and research grants from Takeda Science Foundation and Hoansha Foundation (to TI).

Bough, K. J., Wetherington, J., Hassel, B., Pare, J. F., Gawryluk, J. W., Greene, J. G., et al. (2006). Mitochondrial biogenesis in the anticonvulsant mechanism of the ketogenic diet. Ann. Neurol. 60, 223-235. doi: 10.1002/ana.20899

Caraballo, R. H., Cersósimo, R. O., Sakr, D., Cresta, A., Escobal, N., and Fejerman, N. (2005). Ketogenic diet in patients with Dravet syndrome. Epilepsia 46, 1539-1544. doi: 10.1111/j.1528-1167.2005.05705.x

Catterall, W. A., Leal, K., and Nanou, E. (2013). Calcium channels and short-term synaptic plasticity. J. Biol. Chem. 288, 10742-10749. doi: 10.1074/jbc.R112. 411645

Chang, P., Augustin, K., Boddum, K., Williams, S., Sun, M., Terschak, J. A., et al. (2016). Seizure control by decanoic acid through direct AMPA receptor inhibition. Brain 139, 431-443. doi: 10.1093/brain/awv325

Chang, P., Terbach, N., Plant, N., Chen, P. E., Walker, M. C., and Williams, R. S. (2013). Seizure control by ketogenic diet-associated medium chain fatty acids. Neuropharmacology 69, 105-114. doi: 10.1016/j.neuropharm.2012.11.004

Chiron, C., Marchand, M. C., Tran, A., Rey, E., d'Athis, P., Vincent, J., et al. (2000). Stiripentol in severe myoclonic epilepsy in infancy: a randomised 
placebo-controlled syndrome-dedicated trial. STICLO study group. Lancet 356, 1638-1642. doi: 10.1016/s0140-6736(00)03157-3

Ciarlone, S. L., Grieco, J. C., D’Agostino, D. P., and Weeber, E. J. (2016). Ketone ester supplementation attenuates seizure activity and improves behavior and hippocampal synaptic plasticity in an Angelman syndrome mouse model. Neurobiol. Dis. 96, 38-46. doi: 10.1016/j.nbd.2016.08.002

D’Agostino, D. P., Pilla, R., Held, H. E., Landon, C. S., Puchowicz, M., Brunengraber, H., et al. (2013). Therapeutic ketosis with ketone ester delays central nervous system oxygen toxicity seizures in rats. Am. J. Physiol. Regul. Integr. Comp. Physiol. 304, R829-R836. doi: 10.1152/ajpregu.00506.2012

Dunwiddie, T. V., and Masino, S. A. (2001). The role and regulation of adenosine in the central nervous system. Annu. Rev. Neurosci. 24, 31-55. doi: 10.1146/annurev.neuro.24.1.31

Fenoglio-Simeone, K. A., Wilke, J. C., Milligan, H. L., Allen, C. N., Rho, J. M., and Maganti, R. K. (2009). Ketogenic diet treatment abolishes seizure periodicity and improves diurnal rhythmicity in epileptic Kcna1-null mice. Epilepsia 50, 2027-2034. doi: 10.1111/j.1528-1167.2009.02163.x

Foley, J., Burnham, V., Tedoldi, M., Danial, N. N., and Yellen, G. (2018). BAD knockout provides metabolic seizure resistance in a genetic model of epilepsy with sudden unexplained death in epilepsy. Epilepsia 59, e1-e4. doi: 10.1111/epi.13960

Freeman, J. M., Vining, E. P., Pillas, D. J., Pyzik, P. L., Casey, J. C., and Kelly, L. M. (1998). The efficacy of the ketogenic diet-1998: a prospective evaluation of intervention in 150 children. Pediatrics 102, 1358-1363. doi: 10.1542/peds. 102. 6.1358

Fremeau, R. T. Jr., Voglmaier, S., Seal, R. P., and Edwards, R. H. (2004). VGLUTs define subsets of excitatory neurons and suggest novel roles for glutamate. Trends Neurosci. 27, 98-103. doi: 10.1016/j.tins.2003.11.005

French, J. A., Krauss, G. L., Biton, V., Squillacote, D., Yang, H., Laurenza, A., et al. (2012). Adjunctive perampanel for refractory partial-onset seizures: randomized phase III study 304. Neurology 79, 589-596. doi: 10.1212/WNL. 0b013e3182635735

Giménez-Cassina, A., and Danial, N. N. (2015). Regulation of mitochondrial nutrient and energy metabolism by BCL-2 family proteins. Trends Endocrinol. Metab. 26, 165-175. doi: 10.1016/j.tem.2015.02.004

Giménez-Cassina, A., Martínez-François, J. R., Fisher, J. K., Szlyk, B., Polak, K., Wiwczar, J., et al. (2012). BAD-dependent regulation of fuel metabolism and $\mathrm{K}_{\mathrm{ATP}}$ channel activity confers resistance to epileptic seizures. Neuron 74, 719-730. doi: 10.1016/j.neuron.2012.03.032

Gouder, N., Fritschy, J. M., and Boison, D. (2003). Seizure suppression by adenosine $A_{1}$ receptor activation in a mouse model of pharmacoresistant epilepsy. Epilepsia 44, 877-885. doi: 10.1046/j.1528-1157.2003.03603.x

Haidukewych, D., Forsythe, W. I., and Sills, M. (1982). Monitoring octanoic and decanoic acids in plasma from children with intractable epilepsy treated with medium-chain triglyceride diet. Clin. Chem. 28, 642-645.

Hanada, T., Hashizume, Y., Tokuhara, N., Takenaka, O., Kohmura, N., Ogasawara, A., et al. (2011). Perampanel: a novel, orally active, noncompetitive AMPA-receptor antagonist that reduces seizure activity in rodent models of epilepsy. Epilepsia 52, 1331-1340. doi: 10.1111/j.1528-1167.2011.03109.x

Huttenlocher, P. R. (1976). Ketonemia and seizures: metabolic and anticonvulsant effects of two ketogenic diets in childhood epilepsy. Pediatr. Res. 10, 536-540. doi: 10.1203/00006450-197605000-00006

Huttenlocher, P. R., Wilbourn, A. J., and Signore, J. M. (1971). Medium-chain triglycerides as a therapy for intractable childhood epilepsy. Neurology 21, 1097-1103. doi: 10.1212/WNL.21.11.1097

Juge, N., Gray, J. A., Omote, H., Miyaji, T., Inoue, T., Hara, C., et al. (2010). Metabolic control of vesicular glutamate transport and release. Neuron 68, 99-112. doi: 10.1016/j.neuron.2010.09.002

Kadowaki, A., Sada, N., Juge, N., Wakasa, A., Moriyama, Y., and Inoue, T. (2017). Neuronal inhibition and seizure suppression by acetoacetate and its analog, 2phenylbutyrate. Epilepsia 58, 845-857. doi: 10.1111/epi.13718

Kawamura, M. Jr., Ruskin, D. N., Geiger, J. D., Boison, D., and Masino, S. A. (2014). Ketogenic diet sensitizes glucose control of hippocampal excitability. J. Lipid Res. 55, 2254-2260. doi: 10.1194/jlr.m046755

Kawamura, M. Jr., Ruskin, D. N., and Masino, S. A. (2010). Metabolic autocrine regulation of neurons involves cooperation among pannexin hemichannels, adenosine receptors and $\mathrm{K}_{\mathrm{ATP}}$ channels. J. Neurosci. 30, 3886-3895. doi: 10.1523/JNEUROSCI.0055-10.2010
Kesl, S. L., Poff, A. M., Ward, N. P., Fiorelli, T. N., Ari, C., Van Putten, A. J., et al. (2016). Effects of exogenous ketone supplementation on blood ketone, glucose, triglyceride and lipoprotein levels in Sprague-Dawley rats. Nutr. Metab. 13:9. doi: 10.1186/s12986-016-0069-y

Klein, P., Tyrlikova, I., and Mathews, G. C. (2014). Dietary treatment in adults with refractory epilepsy: a review. Neurology 83, 1978-1985. doi: 10.1212/WNL. 0000000000001004

Kossoff, E. H., and McGrogan, J. R. (2005). Worldwide use of the ketogenic diet. Epilepsia 46, 280-289. doi: 10.1111/j.0013-9580.2005.42704.x

Kovács, Z., D’Agostino, D. P., Dobolyi, A., and Ari, C. (2017). Adenosine A1 receptor antagonism abolished the anti-seizure effects of exogenous ketone supplementation in Wistar Albino Glaxo Rijswijk rats. Front. Mol. Neurosci. 10:235. doi: 10.3389/fnmol.2017.00235

Krauss, G. L., Serratosa, J. M., Villanueva, V., Endziniene, M., Hong, Z., French, J., et al. (2012). Randomized phase III study 306: adjunctive perampanel for refractory partial-onset seizures. Neurology 78, 1408-1415. doi: 10.1212/WNL. 0b013e318254473a

Kwan, P., and Brodie, M. J. (2000). Early identification of refractory epilepsy. $N$. Engl. J. Med. 342, 314-319. doi: 10.2165/00128413-200012240-00036

Larrabee, M. G. (1995). Lactate metabolism and its effects on glucose metabolism in an excised neural tissue. J. Neurochem. 64, 1734-1741. doi: 10.1046/j.14714159.1995.64041734.x

Lee, R. W. Y., Corley, M. J., Pang, A., Arakaki, G., Abbott, L., Nishimoto, M., et al. (2018). A modified ketogenic gluten-free diet with MCT improves behavior in children with autism spectrum disorder. Physiol. Behav. 188, 205-211. doi: 10.1016/j.physbeh.2018.02.006

Liu, H., Yang, Y., Wang, Y., Tang, H., Zhang, F., Zhang, Y., et al. (2018). Ketogenic diet for treatment of intractable epilepsy in adults: a meta-analysis of observational studies. Epilepsia Open 3, 9-17. doi: 10.1002/epi4.12098

Lutas, A., and Yellen, G. (2013). The ketogenic diet: metabolic influences on brain excitability and epilepsy. Trends Neurosci. 36, 32-40. doi: 10.1016/j.tins.2012. 11.005

Ma, W., Berg, J., and Yellen, G. (2007). Ketogenic diet metabolites reduce firing in central neurons by opening $\mathrm{K}_{\text {ATP }}$ channels. J. Neurosci. 27, 3618-3625. doi: 10.1523/JNEUROSCI.0132-07.2007

Martínez-François, J. R., Fernández-Agüera, M. C., Nathwani, N., Lahmann, C., Burnham, V. L., Danial, N. N., et al. (2018). BAD and $\mathrm{K}_{\mathrm{ATP}}$ channels regulate neuron excitability and epileptiform activity. Elife 7:e32721. doi: 10.7554/elife. 32721

Masino, S. A., Li, T., Theofilas, P., Sandau, U. S., Ruskin, D. N., Fredholm, B. B., et al. (2011). A ketogenic diet suppresses seizures in mice through adenosine $\mathrm{A}_{1}$ receptors. J. Clin. Invest. 121, 2679-2683. doi: 10.1172/jci57813

Meldrum, B. S., and Rogawski, M. A. (2007). Molecular targets for antiepileptic drug development. Neurotherapeutics 4, 18-61. doi: 10.1016/j.nurt.2006.11.010

Merritt, H. H., and Putnam, T. J. (1938). Sodium diphenyl hydantoinate in the treatment of convulsive disorders. JAMA 111, 1068-1073. doi: 10.1001/jama. 1938.02790380010004

Neal, E. G., Chaffe, H., Schwartz, R. H., Lawson, M. S., Edwards, N., Fitzsimmons, G., et al. (2008). The ketogenic diet for the treatment of childhood epilepsy: a randomised controlled trial. Lancet Neurol. 7, 500-506. doi: 10.1016/s1474-4422(08)70092-9

Neal, E. G., Chaffe, H., Schwartz, R. H., Lawson, M. S., Edwards, N., Fitzsimmons, G., et al. (2009). A randomized trial of classical and mediumchain triglyceride ketogenic diets in the treatment of childhood epilepsy. Epilepsia 50, 1109-1117. doi: 10.1111/j.1528-1167.2008.01870.x

Oldendorf, W. H. (1973). Carrier-mediated blood-brain barrier transport of short-chain monocarboxylic organic acids. Am. J. Physiol. 224, 1450-1453. doi: 10.1152/ajplegacy.1973.224.6.1450

Parsons, M. P., and Hirasawa, M. (2010). ATP-sensitive potassium channelmediated lactate effect on orexin neurons: implications for brain energetics during arousal. J. Neurosci. 30, 8061-8070. doi: 10.1523/jneurosci.5741-09.2010

Quilichini, P. P., Chiron, C., Ben-Ari, Y., and Gozlan, H. (2006). Stiripentol, a putative antiepileptic drug, enhances the duration of opening of $\mathrm{GABA}_{\mathrm{A}}$ receptor channels. Epilepsia 47, 704-716. doi: 10.1111/j.1528-1167.2006. 00497.x

Reger, M. A., Henderson, S. T., Hale, C., Cholerton, B., Baker, L. D., Watson, G. S., et al. (2004). Effects of $\beta$-hydroxybutyrate on cognition in memory-impaired adults. Neurobiol. Aging 25, 311-314. doi: 10.1016/S0197-4580(03)00087-3 
Rho, J. M. (2017). How does the ketogenic diet induce anti-seizure effects? Neurosci. Lett. 637, 4-10. doi: 10.1016/j.neulet.2015.07.034

Rouach, N., Koulakoff, A., Abudara, V., Willecke, K., and Giaume, C. (2008). Astroglial metabolic networks sustain hippocampal synaptic transmission. Science 322, 1551-1555. doi: 10.1126/science.1164022

Ruskin, D. N., Murphy, M. I., Slade, S. L., and Masino, S. A. (2017). Ketogenic diet improves behaviors in a maternal immune activation model of autism spectrum disorder. PLoS One 12:e0171643. doi: 10.1371/journal.pone.0171643

Ruskin, D. N., Svedova, J., Cote, J. L., Sandau, U., Rho, J. M., Kawamura, M. Jr., et al. (2013). Ketogenic diet improves core symptoms of autism in BTBR mice. PLoS One 8:e65021. doi: 10.1371/journal.pone.0065021

Sada, N., Lee, S., Katsu, T., Otsuki, T., and Inoue, T. (2015). Targeting LDH enzymes with a stiripentol analog to treat epilepsy. Science 347, 1362-1367. doi: $10.1126 /$ science.aaa1299

Sakauchi, M., Oguni, H., Kato, I., Osawa, M., Hirose, S., Kaneko, S., et al. (2011). Retrospective multiinstitutional study of the prevalence of early death in Dravet syndrome. Epilepsia 52, 1144-1149. doi: 10.1111/j.1528-1167.2011.03053.x

Sills, M. A., Forsythe, W. I., and Haidukewych, D. (1986). Role of octanoic and decanoic acids in the control of seizures. Arch. Dis. Child. 61, 1173-1177. doi: 10.1136/adc.61.12.1173

Simeone, K. A., Matthews, S. A., Rho, J. M., and Simeone, T. A. (2016). Ketogenic diet treatment increases longevity in Kcnal-null mice, a model of sudden unexpected death in epilepsy. Epilepsia 57, e178-e182. doi: 10.1111/epi.13444

Simeone, T. A., Simeone, K. A., Stafstrom, C. E., and Rho, J. M. (2018). Do ketone bodies mediate the anti-seizure effects of the ketogenic diet? Neuropharmacology 133, 233-241. doi: 10.1016/j.neuropharm.2018.01.011

Smith, D., Pernet, A., Hallett, W. A., Bingham, E., Marsden, P. K., and Amiel, S. A. (2003). Lactate: a preferred fuel for human brain metabolism in vivo. J. Cereb. Blood Flow Metab. 23, 658-664. doi: 10.1097/01.wcb.0000063991.19746.11

Tanner, G. R., Lutas, A., Martínez-François, J. R., and Yellen, G. (2011). Single $\mathrm{K}_{\mathrm{ATP}}$ channel opening in response to action potential firing in mouse dentate granule neurons. J. Neurosci. 31, 8689-8696. doi: 10.1523/jneurosci.5951-10. 2011

Trojnar, M. K., Wojtal, K., Trojnar, M. P., and Czuczwar, S. J. (2005). Stiripentol. A novel antiepileptic drug. Pharmacol. Rep. 57, 154-160.

Vanitallie, T. B., Nonas, C., Di Rocco, A., Boyar, K., Hyams, K., and Heymsfield, S. B. (2005). Treatment of parkinson disease with diet-induced hyperketonemia: a feasibility study. Neurology 64, 728-730. doi: 10.1212/01. wnl.0000152046.11390.45

Wheless, J. W. (2008). History of the ketogenic diet. Epilepsia 49, 3-5. doi: 10.1111/j.1528-1167.2008.01821.x

Won, Y. J., Lu, V. B., Puhl, H. L. III., and Ikeda, S. R. (2013). $\beta$-Hydroxybutyrate modulates $\mathrm{N}$-type calcium channels in rat sympathetic neurons by acting as an agonist for the G-protein-coupled receptor FFA3. J. Neurosci. 33, 19314-19325. doi: 10.1523/JNEUROSCI.3102-13.2013

Zawar, C., Plant, T. D., Schirra, C., Konnerth, A., and Neumcke, B. (1999). Cell-type specific expression of ATP-sensitive potassium channels in the rat hippocampus. J. Physiol. 514, 327-341.

Zucker, R. S., and Regehr, W. G. (2002). Short-term synaptic plasticity. Annu. Rev. Physiol. 64, 355-405. doi: 10.1146/annurev.physiol.64.092501. 114547

Conflict of Interest Statement: The authors declare that the research was conducted in the absence of any commercial or financial relationships that could be construed as a potential conflict of interest.

Copyright (c) 2018 Sada and Inoue. This is an open-access article distributed under the terms of the Creative Commons Attribution License (CC BY). The use, distribution or reproduction in other forums is permitted, provided the original author(s) and the copyright owner(s) are credited and that the original publication in this journal is cited, in accordance with accepted academic practice. No use, distribution or reproduction is permitted which does not comply with these terms. 